\title{
Retornos da Educação e o Desequilíbrio Regional no Brasil
}

\author{
Carlos Alberto Manso*, Flávio Ataliba Barreto ${ }^{\dagger}$, João Mário S. de \\ França ${ }^{\ddagger}$
}

Conteúdo: 1. Introdução; 2. Fatos Estilizados; 3. Comparando a Evolução do Bem-Estar Social entre as Regiões; 4. Decompondo os Ganhos de Renda no Mercado de Trabalho entre as Regiões; 5. Considerações Finais.

Palavras-chave: Desequilíbrio Regional, Bem-Estar Social, Mercado de Trabalho.

Códigos JEL: $\quad$ D31, J21, I32.

O problema do desequilíbrio regional brasileiro normalmente tem sido tratado na literatura nacional a partir de investigações que utilizam a renda e/ou PIB per capita como variáveis de análise. Para sociedades com baixos níveis de desigualdade, esse procedimento é uma boa representação do bem-estar social. Entretanto, para economias com elevados níveis de pobreza e desigualdade, a utilização da renda ou PIB pode não ser apropriada. Nesse sentido, esse artigo discute se a aproximação da renda per capita existente entre o Nordeste e o Sudeste brasileiro também vem ocorrendo em termos de bem-estar social. Para esse objetivo, duas medidas de bem-estar social são utilizadas: Sen (1977) e Kakwani e Son (2008). Os resultados apontam que, assim como ocorre com a renda per capita, também está havendo aproximação de bemestar considerando a medida de Sen. Entretanto, quando se leva em conta o movimento da renda dos mais pobres, presente no segundo índice, constata-se um afastamento entre as duas regiões. As análises são feitas para o período de 1995 a 2007. Por fim, identificou-se que foram os ganhos de produtividade dos trabalhadores pertencentes a famílias pobres no Sudeste os principais responsáveis por esse distanciamento.

The problem of Brazilian regional disequilibrium has usually been treated in the national literature from investigations using income and/or GDP per capita as a variable of analysis. For communities with low levels of inequality, this procedure is a good representation of the social welfare. However, for economies with high levels of poverty and inequality, the use of income

\footnotetext{
*Universidade Federal do Ceará (CAEN/UFC). E-mail: calbertomanso@yahoo.com.br

†Universidade Federal do Ceará (CAEN/UFC). E-mail: ataliba@ufc.br

${ }^{\ddagger}$ Universidade Federal do Ceará (CAEN/UFC). E-mail: joao.franca@uf c.br
} 
or GDP cannot be appropriate. Accordingly, this article discusses if the approximation of per capita income between the Northeast and Southeast Brazil has also occurred in terms of social welfare. To this, two measures of social welfare are used: Sen (1977) and Kakwani e Son (2008). The results suggest as occurs with per capita income, there has also been assimilation of welfare, considering Sen's proposal. However, when it is considered the movement of the poorest income, present in the second index, there is a distance between the two regions. The analyses are made for the period 1995 to 2007. Finally, it was identified that the productivity gains of workers belonging to poor families in the Southeast were the main responsible for this gap.

\section{INTRODUÇÃO}

O problema do desequilíbrio regional no Brasil e a ideia da convergência entre as regiões têm sempre sido temas de grande interesse na pesquisa acadêmica no país e estão na maioria das vezes no centro das preocupações das ações públicas. De uma forma geral, as evidências apontam a existência de um processo, mesmo que lento, de aproximação entre os diversos estados brasileiros (Ferreira e Diniz, 1995, Ellery Jr. e Ferreira, 1996, Ferreira, 1996, 1999, 2000, Zini Jr., 1998). No entanto, trabalhos mais recentes como Mossi et alii (2003), Andrade et alii (2004) e Gondim et alii (2007) sugerem que essa tendência vem ocorrendo em termos de clusters espaciais em que um grupo de baixa renda seria representado pelas regiões Nordeste e Norte e um outro, de renda mais elevada, pelas regiões Sudeste, Sul e Centro-Oeste. Essas evidências assemelham-se ao que vem ocorrendo em nível mundial, como sugerido por Jones (1997).

A literatura nacional tem apontado que grande parte do diferencial de renda entre as regiões tem sido explicada pelas disparidades educacionais (Barros, 1993, Barros e Mendonça, 1995, 1997, Pessoa, 2001, Pessoa et alii, 2007). A título de exemplo, Salvato et alii (2007), através de exercícios contrafactuais para o ano de 2005 , mostra que mais de $50 \%$ do diferencial da renda do trabalho entre o Nordeste e 0 Sudeste são explicados pelas diferenças de escolaridade.

A despeito da importância que a renda per capita possa ter na análise de convergência e, por consequência, sobre considerações de bem-estar, esse tipo de investigação torna-se menos relevante quando os níveis de desigualdade e pobreza numa economia são muito elevados e sofrem grandes variações no período de investigação. Como bem documentado em Barros et alii (2006) e Neri (2006), a desigualdade no Brasil passa por um processo de significante declínio desde 2001, mas apesar desse progresso, dos 126 países para os quais existem estatísticas sobre o coeficiente de Gini, $90 \%$ têm distribuição menos concentrada do que o Brasil.

Por outro lado, essa situação é agravada quando se examina o problema da desigualdade na perspectiva regional. Comparando-se Nordeste e Sudeste, constata-se que, em 2001, a primeira região possuía quase $60 \%$ dos seus habitantes no terço inferior da renda - o que representa $49,2 \%$ da população de todo país - enquanto que, no Sudeste, apenas $21,4 \%$ da população pertencia a essa camada inferior de renda (IPEA, 2008). Ademais, como se verá a seguir, a trajetória de queda da desigualdade e da pobreza entre essas regiões também ocorre de forma distinta. Essas evidências jogam luz na ideia de que, ao se examinar a existência de convergência de renda per capita entre regiões, é importante que se adicionem outras preocupações. Como bem lembrado em Wodon e Yitzhaki (2005), o crescimento da renda e a hipótese de convergência somente teriam sentido se acompanhados de aumentos nos níveis de bem-estar e, no caso de sociedades muito desiguais, é premente a necessidade de se ponderar de forma distinta a evolução da renda entre as diversas camadas sociais. 
Um ingrediente adicional nesse debate surge quando se pretende analisar o problema da aproximação de renda entre regiões em períodos de expansão e contração da atividade econômica nacional. Nesse sentido, se as regiões de um dado país possuem dinâmicas distintas, pode-se imaginar que as flutuações de renda nacional no curto prazo tenham rebatimentos distintos em cada localidade, não só em termos de renda, mas também no que concerne a indicadores de desigualdade, pobreza e bem-estar. Pode-se imaginar, por exemplo, que se uma região tem uma maior influência do mercado na sua atividade econômica, é de se esperar que num período de retração da renda nacional, seus níveis de renda e pobreza sejam mais afetados quando comparados a outra região com menor atividade empresarial.

Assim, dado o diferencial de desigualdade de renda e pobreza existente entre as regiões Nordeste e Sudeste é oportuno verificar como a renda nacional e seus movimentos ocorridos a partir de 1995 impactam o diferencial de renda e bem-estar nas regiões. É evidente que essa discussão passa necessariamente por um bom entendimento do comportamento da renda entre as camadas mais pobres da população, em cada localidade, que por sua vez é consequência, em parte, ao que vem ocorrendo no mercado de trabalho no que diz respeito ao comportamento de indicadores como taxa de desemprego, horas trabalhadas, produtividade e participação da força de trabalho.

Diante desse desafio, este estudo está organizado da seguinte forma: além da seção introdutória, a Seção 2 apresenta os fatos estilizados dos movimentos de renda, desigualdade e pobreza no Brasil, Nordeste e Sudeste a partir de 1995. Na Seção 3, avalia-se a evolução do bem-estar entre as regiões seguindo a metodologia de Sen (1977) e de um índice de crescimento pró-pobre proposto por Kakwani e Son (2008). A decomposição da renda do trabalho, para explicar os movimentos da renda nacional e seus rebatimentos regionais, é realizada na Seção 4. Por fim, a Seção 5 é dedicada às Considerações Finais.

\section{FATOS ESTILIZADOS}

As Figuras 1-3 apresentam a evolução da renda per capita, da desigualdade e da proporção de pobres no Brasil, Sudeste e Nordeste, de 1995 a 2007. Inicialmente, examinando a evolução da renda, destacamse 5 períodos, sendo 3 de expansão dado por 1995-1998, 1999-2002 e 2003-2007 e dois de contração 1998-1999 e 2002-2003, em que o primeiro está relacionado à crise cambial quando da desvalorização do Real e o segundo na eminência de eleição do Presidente Lula, quando o mercado teve receios quanto à manutenção da estabilidade macroeconômica. As taxas de variação em cada período podem ser vistas entre as figuras. Na Figura 1, verifica-se que o Nordeste apresenta taxas de expansão da renda superiores às do Sudeste e Brasil. Nos períodos de contração, a renda do Nordeste retrai-se menos no período 19981999, porém ligeiramente mais no segundo período. De uma forma geral, esses resultados sugerem um movimento de aproximação da renda per capita entre essas regiões nesse período.

Em extensão, a desigualdade de renda medida pelo índice de Gini é analisada na Figura 2. Em todo período, verifica-se que o Nordeste apresenta os maiores níveis. No primeiro período de expansão da renda, a desigualdade no Sudeste reduz-se ligeiramente, enquanto que no Nordeste cresce, mas já no período seguinte (1999-2002) o movimento entre as regiões é inverso. Entretanto, no terceiro período de expansão há uma queda na desigualdade nas duas regiões. Quanto aos períodos de contração da renda nacional verifica-se redução no índice de GINI nas duas regiões: no primeiro período há maior queda na concentração de renda do Sudeste, enquanto que no segundo, a maior redução ocorre no Nordeste. Pode-se observar, segundo esses movimentos, que não existe um padrão comum de comportamento da desigualdade nas fases de expansão ou contração da renda do país. Entretanto, quando se conciliam as informações relativas à evolução da desigualdade com a da renda, percebe-se que em períodos de acelerada expansão, como no último, a desigualdade no Sudeste cai mais fortemente.

O entendimento do padrão de rebatimentos regionais em termos de renda, desigualdade, pobreza e bem-estar, de movimentos da renda nacional é de natureza muito complexa, pois depende de vários cenários. Por exemplo, se o período de expansão da economia brasileira estiver sendo motivado 
Figura 1: Evolução da renda familiar per capita, Brasil e regiões sudeste e nordeste

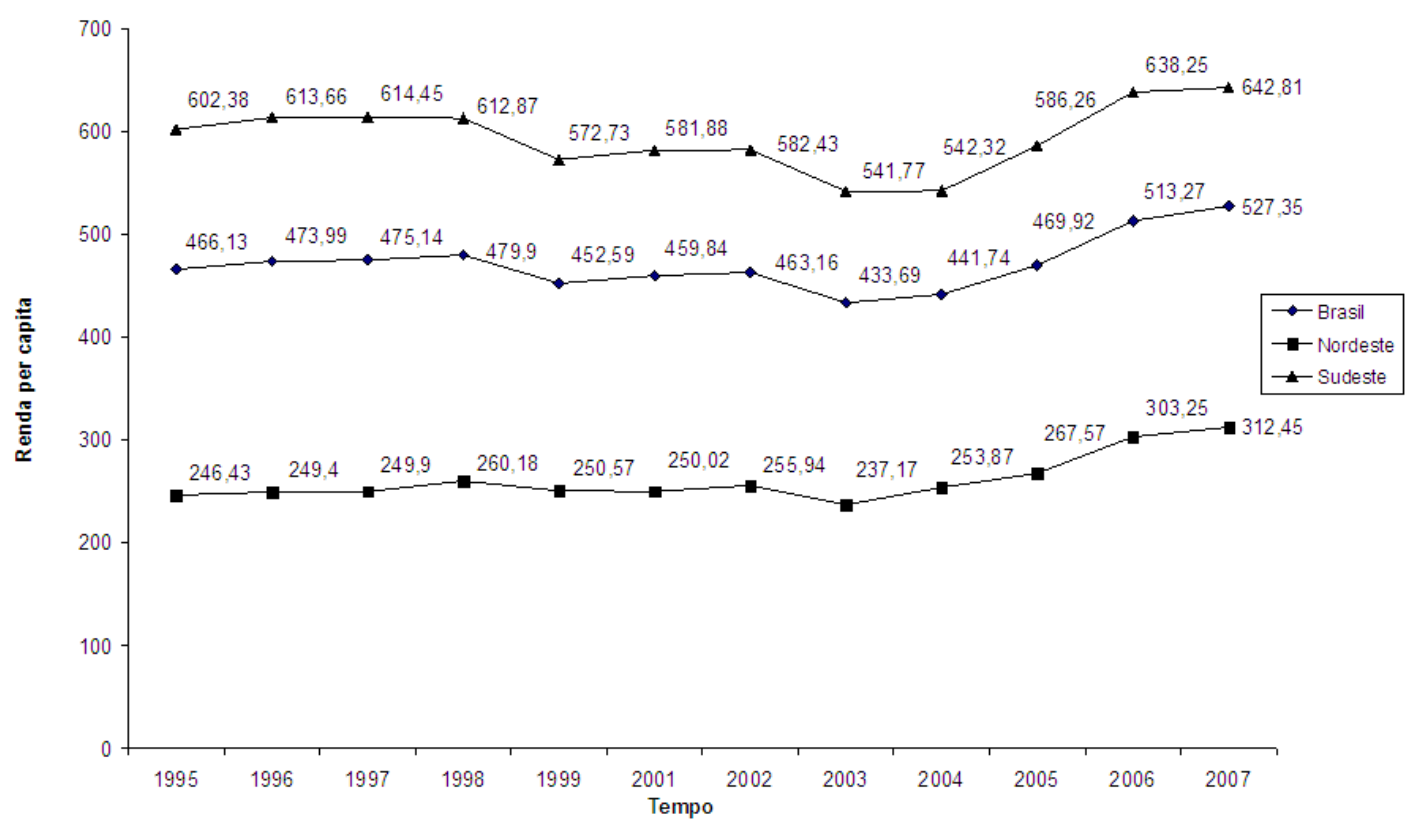

Fonte: Elaboração dos autores a partir dos dados da PNAD/IBGE.

por uma maior demanda de produtos agrícolas, aquelas regiões e setores do mercado de trabalho que estiverem dedicados a essa atividade seriam relativamente mais beneficiados. Isso poderia contribuir para mudanças na composição da renda relativa entre os diversos segmentos, alterando por sua vez os indicadores de desigualdade e pobreza em cada região. Por outro lado, se a retração ocorresse por um aumento nos juros reais na economia, o impacto poderia ser outro, uma vez que aquelas regiões do país e setores que dependessem mais de crédito seriam mais afetadas, com consequências diretas sobre 0 mercado de trabalho nessas localidades. Nesse contexto, a dinâmica da renda pode ter reflexos imediatos e distintos sobre os níveis de desigualdade e pobreza em cada região. A literatura tem sido pródiga em mostrar as inter-relações dessas variáveis (Ravallion, 2001, Bourguignon, 2004, Adams, 2004, Son, 2007, Wal, 2008).

A Figura 3 ilustra o comportamento da pobreza tomando por base o índice da proporção de pobres como reflexo do comportamento da renda e da desigualdade. ${ }^{1}$ Inicialmente, percebe-se que os níveis no Nordeste são bem superiores à média nacional e ao Sudeste. Entretanto, como visto anteriormente, no primeiro período de expansão, o Nordeste apresentou uma variação na renda mais que três vezes a da região Sudeste, mas a redução na proporção de pobres nessa região foi superior em apenas $25 \%$ a que aconteceu no Sudeste. Grande parte desse resultado pode ser explicada pelo movimento da

\footnotetext{
${ }^{1}$ Para esse cálculo, e para todas as outras tabulações de índices de pobreza neste estudo, foram utilizadas as linhas de pobreza regionalizadas presentes em Neri (2007) e deflacionadas pelo INPC, conforme metodologia presente em Corseuil e Foguel (2002).
} 
Figura 2: Evolução da desigualdade de renda familiar per capita, Brasil e regiões nordeste e sudeste

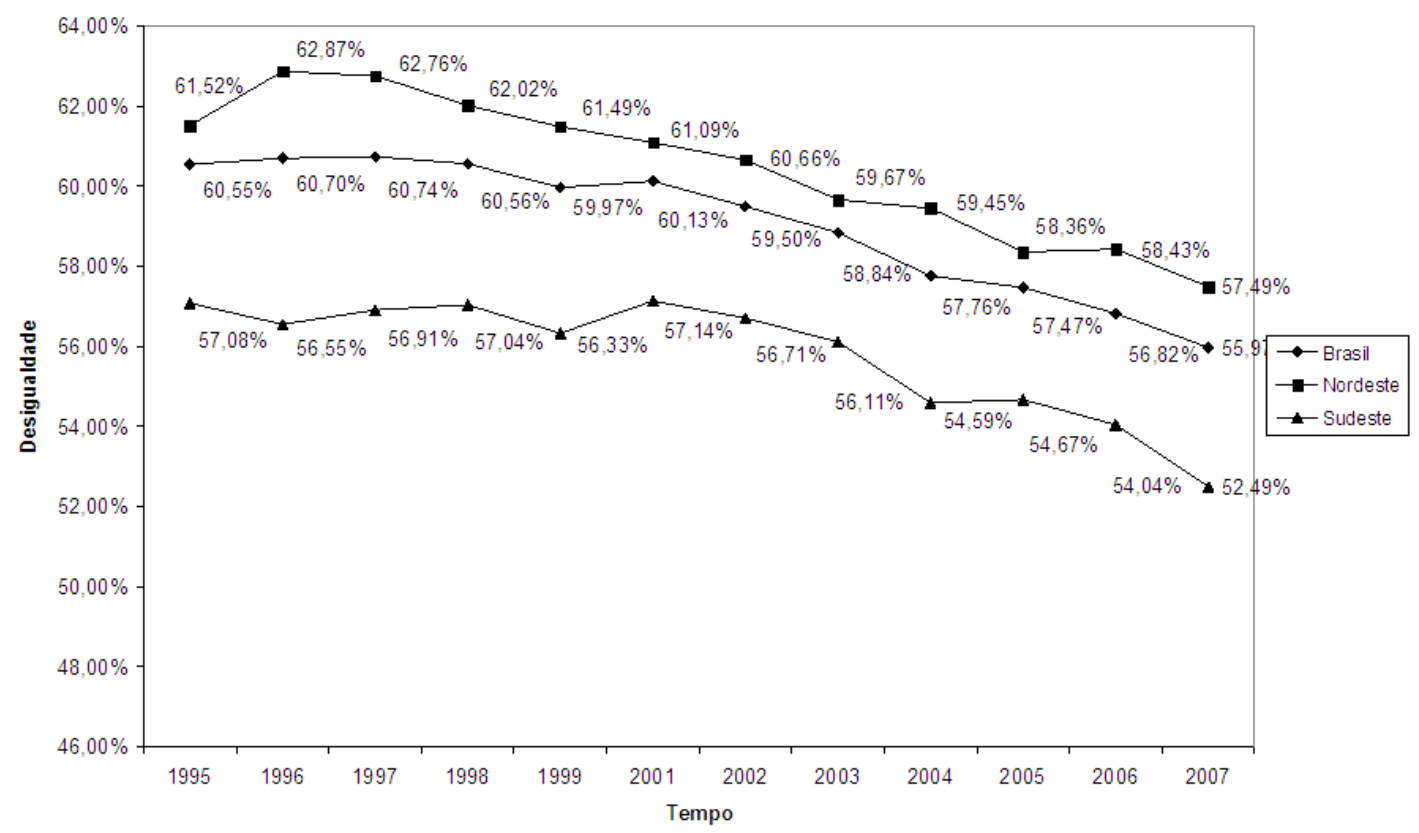

Fonte: Elaboração dos autores a partir dos dados da PNAD/IBGE.

desigualdade, uma vez que enquanto no Nordeste ela teve um acréscimo de $1,13 \%$, na região Sudeste houve uma redução de $-0,08 \%$.

Explicação semelhante pode ser dada para o período seguinte, o de contração, onde a renda no Sudeste caiu de $-6,55 \%$, enquanto que no Nordeste a queda foi menor, de $-3,69 \%$. No entanto, o aumento da pobreza nesse período nessas regiões teve comportamento de certa forma bem semelhante. A redução da desigualdade no Sudeste foi mais expressiva, o que compensou o maior impacto negativo do declínio da renda. Por fim, um exemplo mais emblemático desse processo ocorre no período final de expansão da renda (2003-2007). Nesse caso, o Sudeste conseguiu reduzir mais expressivamente a pobreza do que o Nordeste, $(41,19 \%$ contra $29,6 \%)$, tendo um crescimento bem menor na renda $(18,66 \%$ contra $31,74 \%)$. Novamente, isso se deveu ao comportamento da desigualdade, que teve queda bem mais expressiva no Sudeste.

A Tabela 1 consolida essas informações e duas evidências importantes são levantadas. A primeira é que o Nordeste apresenta, nos períodos de expansão da renda, taxas superiores às do Sudeste e, nos períodos de recessão, elas se reduzem proporcionalmente menos. Isso sugere uma tendência de aproximação entre as duas regiões em termos de renda familiar per capita, fortalecendo a ideia de convergência entre elas. Entretanto, dados os diferenciais nos níveis da desigualdade e pobreza e nas suas dinâmicas, pode ser razoável pensar que os ganhos de renda estejam se traduzindo de forma diferente em termos de bem-estar social, como discutido anteriormente. Nesse sentido, numa primeira etapa é imperativo observar o comportamento do bem-estar social em cada região, de modo a verificar 
Figura 3: Evolução do índice de pobreza FGT(0), Brasil e regiões sudeste e nordeste

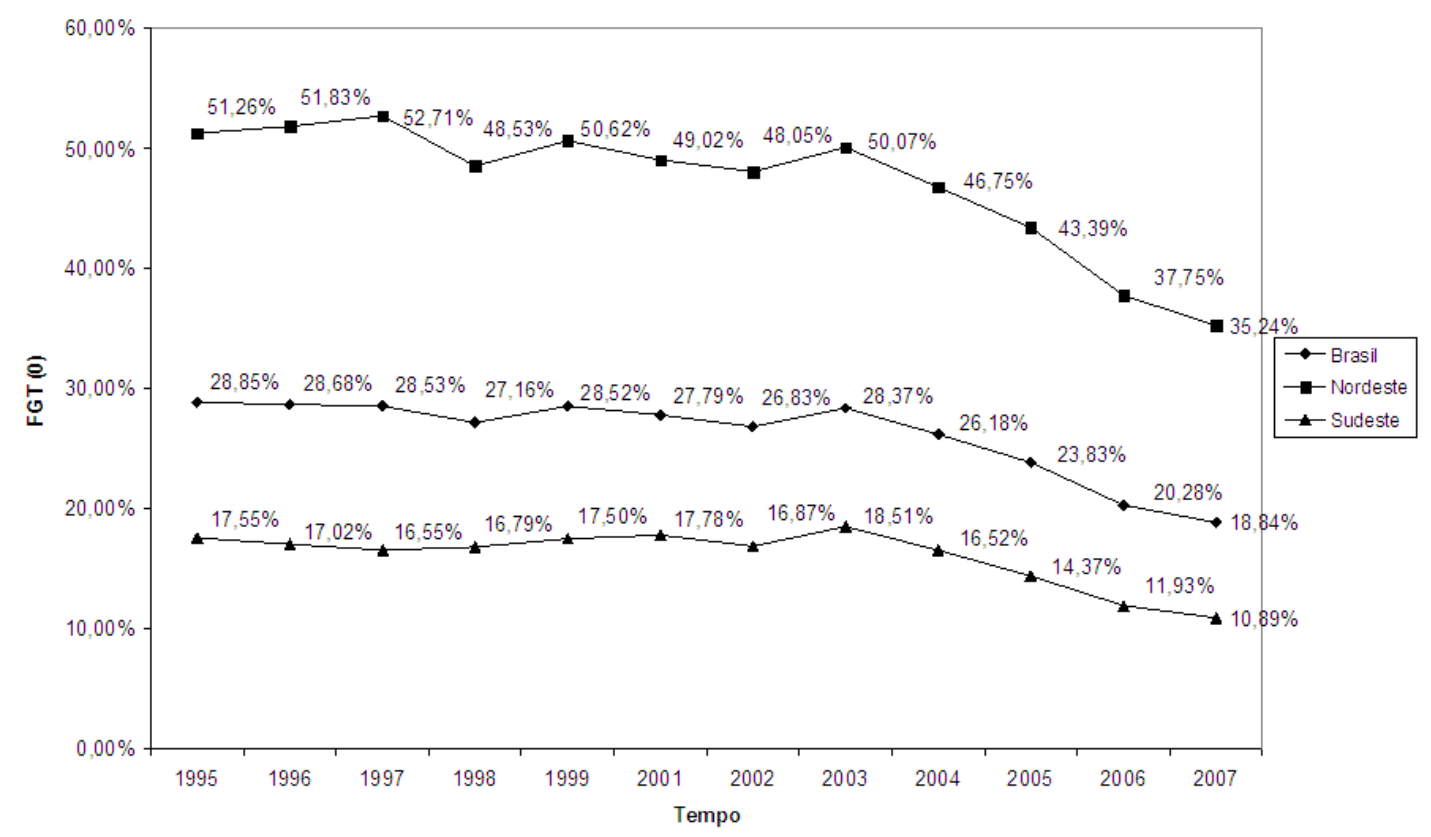

Fonte: Elaboração dos autores a partir dos dados da PNAD/IBGE.

se a tendência de convergência na renda também está se traduzindo em aproximação em termos de bem-estar.

\section{COMPARANDO A EVOLUÇÃO DO BEM-ESTAR SOCIAL ENTRE AS REGIÕES}

Considera-se nessa seção a título de avaliação da evolução do bem-estar entre a região Nordeste e Sudeste dois índices: o índice de Bem-Estar Social de Sen (1977) e uma medida de intensidade de crescimento "pró-pobre" dado por Kakwani e Son (2008), conhecida como Poverty Equivalent Growth Rate (PEGR).

\subsection{0 índice de bem-estar social de Sen}

Sen (1977) constrói um índice de Bem-Estar $(W)$ a partir da seguinte relação entre renda $(\mu)$ e desigualdade $(G)$ :

$$
W=\mu(1-\alpha G)
$$

sendo $\alpha$ um parâmetro de aversão à desigualdade.

Apresentada dessa forma, a medida de Sen representa a renda disponível na sociedade após a subtração dos efeitos da desigualdade, o que implica que as perdas sociais decorrentes da distribuição desigual entre os indivíduos equivalem à distância entre a renda média e o bem-estar social. É calculado esse 


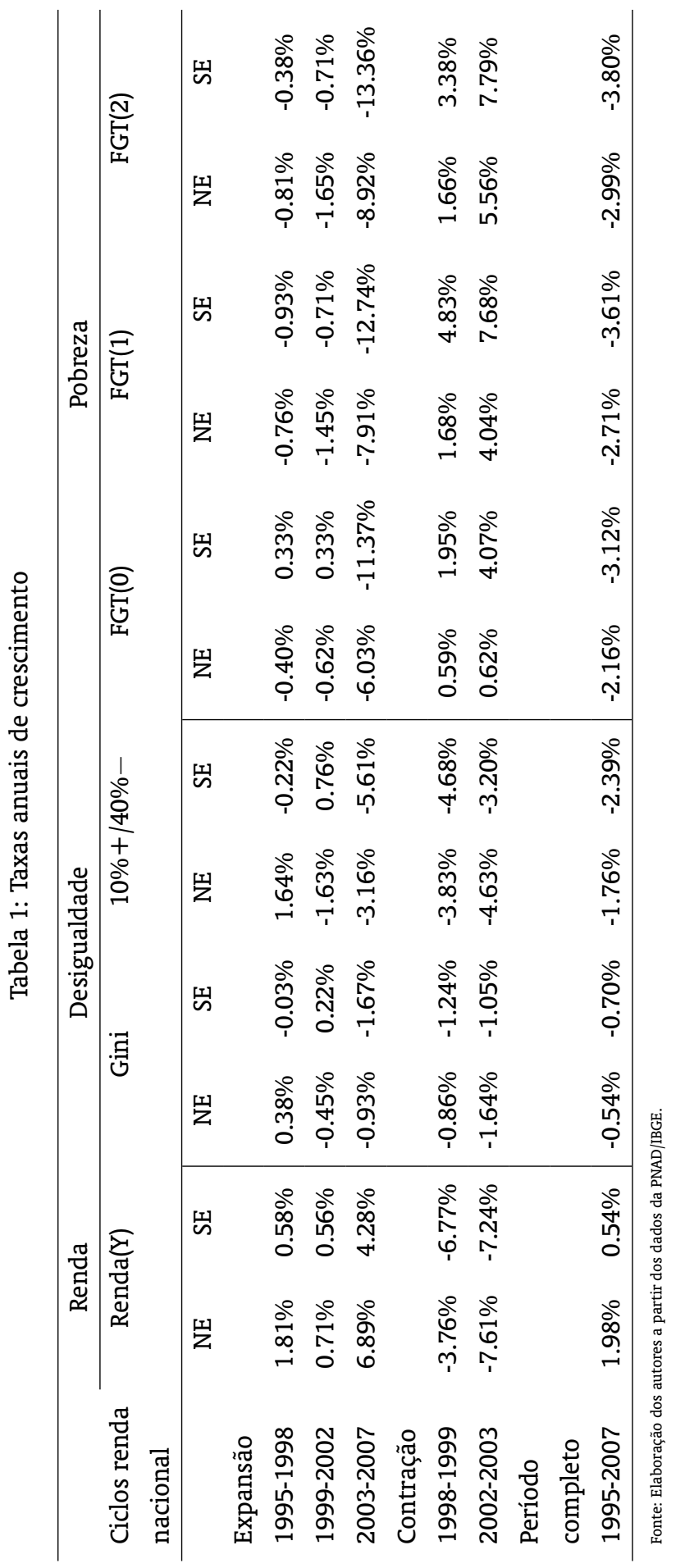


índice a partir da renda familiar per capita tomando o parâmetro de aversão à desigualdade em (1) igual a uma unidade, isto é, $\alpha=1$. Ao supor isso, considera-se uma máxima aversão à concentração de renda. As informações sobre esse índice estão apresentadas na Figura 4.

Figura 4: Evolução do bem-estar de Sen, Brasil e regiões sudeste e nordeste

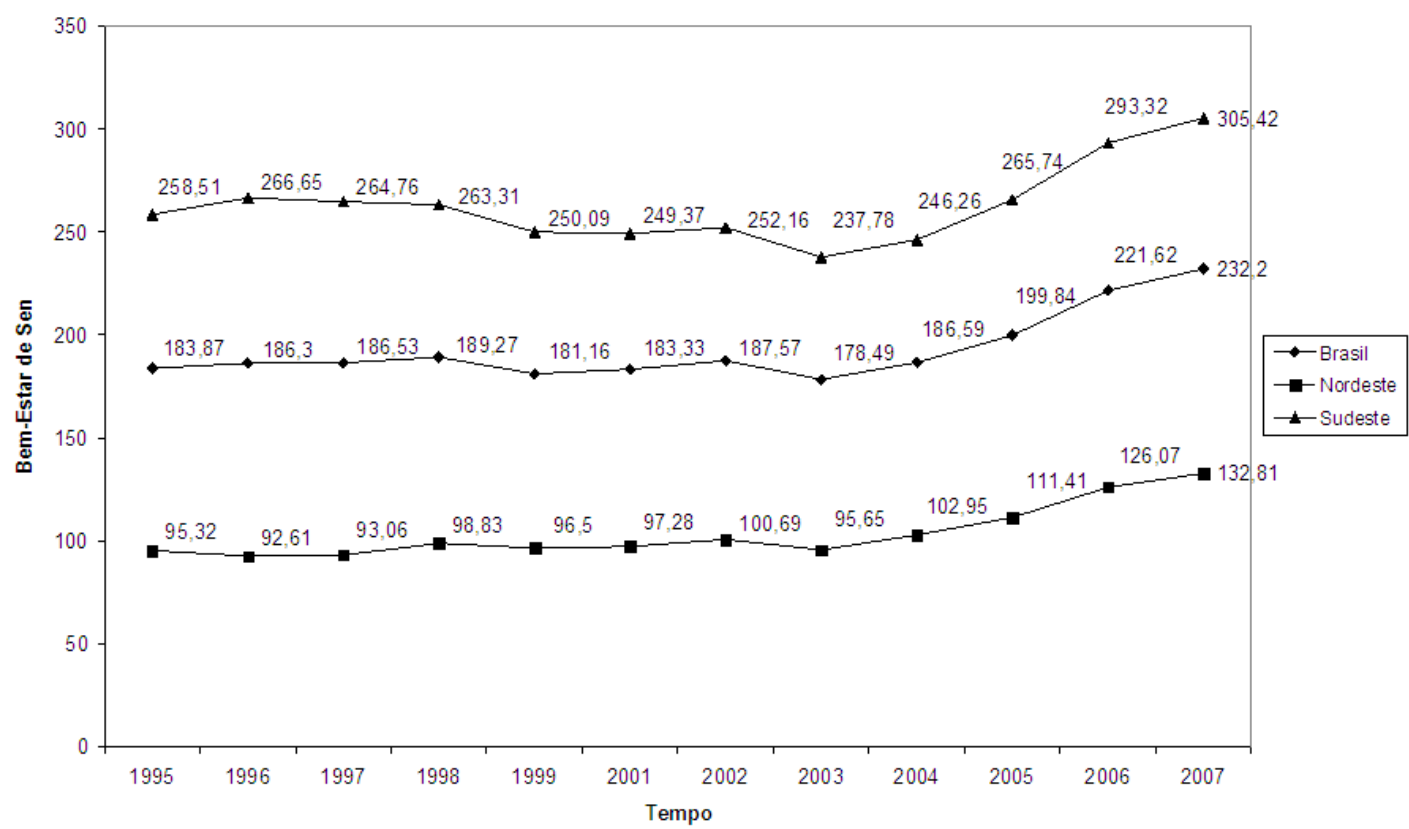

Fonte: Elaboração dos autores a partir dos dados da PNAD/IBGE.

Constata-se inicialmente que o índice de Sen para o Nordeste é sempre inferior ao do Sudeste, em todos esses anos. Isso ocorre tanto pelo fato de seus níveis de renda serem inferiores quanto os níveis de desigualdade, superiores ao Sudeste. Quanto à possibilidade de estar havendo aproximação em termos de bem-estar, a segunda coluna da Tabela 2 apresenta as taxas anuais de crescimento desse índice considerando os períodos de expansão e contração da renda nacional. Como pode ser visto, o Nordeste apresenta taxas superiores às do Sudeste em cada período de expansão. Além do mais, nos períodos de contração, o Nordeste tem menor redução, de modo que, quando se analisa a taxa média de evolução no período completo, essa região teve um ganho de bem-estar de $2,76 \%$ ao ano, sendo quase o dobro do verificado no Sudeste ( $1,39 \%$ a.a).

A justificativa para esse resultado vem do fato da desigualdade em todo o período ter caído menos na região Nordeste $(-0,54 \%)$ que no Sudeste $(-0,70 \%)$ e que, de forma mais importante, a expansão da renda média no Nordeste, de $1,98 \%$, foi bastante superior a do Sudeste com $0,54 \%$ (Tabela 1). Nesse sentido, utilizando esse critério de bem-estar, pode-se afirmar que está havendo aproximação entre as regiões Nordeste e Sudeste semelhante ao que vem ocorrendo em termos de renda familiar per capita. De certa forma, esses resultados corroboram as evidências levantadas em Marinho et alii (2004), que, utilizando o mesmo indicador, apontam que durante a década de 1990 diversos estados nordestinos foram os mais eficientes na geração de bem-estar social. 


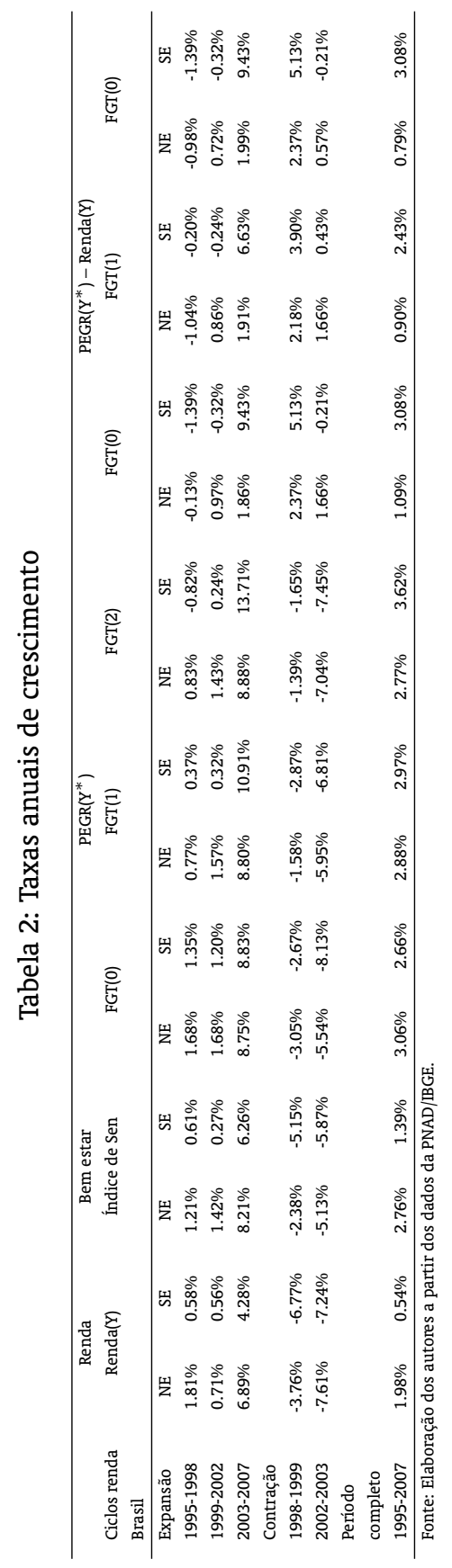


Entretanto, um aspecto importante que se deve levar em conta, em termos do índice de Sen, é que ele pode estar melhorando em parte pela redução da desigualdade, a partir de uma menor participação na renda total das camadas mais ricas da população, assim como pelo aumento da renda média com menor intensidade para os mais pobres. Assim, esses possíveis efeitos, tomados em conjunto, são pouco informativos sobre a evolução da renda nas camadas mais pobres da população. Ademais, se considerarmos que as pessoas com menores rendas deveriam ter um peso maior no cálculo do bemestar, é fundamental que o índice de bem-estar reflita essa necessidade. Para tanto, a metodologia de Kakwani e Son (2008) ajuda-nos a avaliar o movimento da renda e da desigualdade nessa perspectiva, ou seja, através da ideia de um padrão de crescimento da renda em que os benefícios aos mais pobres tenham mais peso relativamente aos não-pobres.

\subsection{A medida da intensidade do crescimento "pró-pobre" de Kakwani e Son}

A ideia da Poverty Equivalente Growth Rate, metodologia presente em Kakwani e Son (2008), procura incorporar os efeitos da expansão da renda nos níveis mais baixos da distribuição, na queda da desigualdade. Assim, ela sugere o cálculo de uma taxa de crescimento hipotética na renda $\left(\gamma^{*}\right)$ que resultaria num nível semelhante de redução da pobreza observado, caso o crescimento da renda média $\gamma$ não tenha sido acompanhado de mudanças na desigualdade. Assim, esse ganho hipotético de renda poderia ser calculado a partir da expressão (2) abaixo:

$$
\gamma^{*}=(\delta / \eta) \gamma
$$

onde $\delta$ é a elasticidade-pobreza total motivada pela expansão da renda média e da desigualdade e $\eta$ elasticidade crescimento-pobreza. Assim, caso $\gamma^{*}>\gamma>0$, a expansão da renda entre os mais pobres seria mais que proporcional ao da renda média da economia o que provocaria evidentemente uma redução da desigualdade. A diferença entre $\gamma^{*}$ e $\gamma$ forneceria a intensidade do crescimento "pró-pobre" ocorrido e por extensão poderia fornecer também uma medida de ganho de "bem-estar social".

Para o cálculo efetivo da PEGR para as medidas FGT de pobreza, utilizamos as rendas médias $\mu_{1}$ e $\mu_{2}$ e as curvas de Lorenz $L_{1}(p)$ e $L_{2}(p)$, respectivamente para os anos iniciais e finais seguiu-se os seguintes passos:

$1^{\circ}$ Determinação do grau de privação $\theta=\int_{0}^{z} P(x) f(x) d x$, onde é uma medida de pobreza de uma distribuição de rendimentos $x$ de frequência $f(x)$. Para tanto, utilizam-se as linhas de pobreza $z$ regionalizadas presentes em Neri (2007). Estas linhas são deflacionadas pelo INPC, conforme metodologia presente em Corseuil e Foguel (2002).

$2^{\circ}$ Cálculo da taxa de crescimento da renda média por: $\gamma=\operatorname{Ln}\left(\mu_{2}\right)-\operatorname{Ln}\left(\mu_{1}\right)$.

$3^{\circ}$ A partir dos valores do índice de Pobreza nos anos iniciais e finais do período, $\theta\left(z, \mu_{1}, L_{1}(p)\right)$ e $\theta\left(z, \mu_{2}, L_{2}(p)\right)$, e de valores contrafactuais dessa medida, $\theta\left(z, \mu_{2}, L_{1}(p)\right)$ e $\theta\left(z, \mu_{1}, L_{2}(p)\right)$, obtidos com renda média de um ano e curva de Lorenz de outro, calculam-se as estimativas para:

$$
\begin{aligned}
\eta & =\frac{1}{2}\left[\operatorname { l n } \left(\theta\left(z, \mu_{2}, L_{1}(p)\right)-\ln \left(\theta\left(z, \mu_{1}, L_{1}(p)\right)+\ln \left(\theta\left(z, \mu_{2}, L_{2}(p)\right)-\ln \left(\theta\left(z, \mu_{1}, L_{2}(p)\right)\right] / \gamma \quad\right. \text { e }\right.\right.\right. \\
\zeta & =\frac{1}{2}\left[\operatorname { l n } \left(\theta\left(z, \mu_{1}, L_{2}(p)\right)-\ln \left(\theta\left(z, \mu_{1}, L_{1}(p)\right)+\ln \left(\theta\left(z, \mu_{2}, L_{2}(p)\right)-\ln \left(\theta\left(z, \mu_{2}, L_{1}(p)\right)\right] / \gamma\right.\right.\right.\right.
\end{aligned}
$$

$4^{\circ}$ Cálculo do valor total da elasticidade-pobreza $(\delta)$ pela soma entre as variáveis $\eta$ e $\zeta$.

$5^{\circ}$ Cálculo do valor da PEGR, seguindo: $\gamma^{*}=(\delta / \eta) \gamma$. 
Pode-se observar que para o cálculo das elasticidades acima foi considerada a elasticidade no arco, ou seja, ela é tomada na média em dois períodos tanto para os valores de renda como para a curva de Lorenz. Esse procedimento evita que o ano inicial ou terminal possa influenciar de forma significativa seus valores, o que poderia sub ou superestimar os valores da renda hipotética gerada, levando à conclusões incorretas. $^{2}$

O cálculo das PEGR para as regiões Nordeste e Sudeste estão apresentados na Tabela 2. Para os períodos de expansão até 2002, a variação na PEGR no Nordeste é superior à do Sudeste para todos os indicadores de pobreza utilizados. Isso significa que, se o crescimento econômico não tivesse sido acompanhado de mudanças na desigualdade, a taxa de crescimento da renda que resultaria no mesmo nível de redução da pobreza de $\gamma$, seria maior na primeira região do que na segunda, o que seria um indicativo de maior bem-estar social. No entanto, para o terceiro período de expansão (2003-2007), a PEGR do Sudeste passa a ser maior em cada índice. Nos períodos de contração, com exceção de $\mathrm{P}(0)$ para 1998-1999, a PEGR no Sudeste é mais afetada. No período completo, apenas a PEGR para FGT(0) é superior no Nordeste já que para FGT(1) e FGT(2) o crescimento da renda média é mais intenso no Sudeste.

As três últimas colunas da Tabela 2 apresentam a diferença entre $\left(\gamma^{*}-\gamma\right)$ para as medidas FGT. Como pode ser visto, no primeiro período de expansão verifica-se que $0<\gamma^{*}<\gamma$ nas duas regiões, ou seja, há aumento na renda média da economia com redução da pobreza, mas esse movimento é acompanhado pelo aumento da desigualdade. Quanto ao segundo período de expansão, ele é semelhante ao primeiro para o Sudeste, mas para o Nordeste há um ganho de renda mais que proporcional entre os mais pobres. Entre 2003-2007, verifica-se novamente ganhos de renda mais que proporcional para as camadas mais baixas da população nas duas regiões, mas é mais intenso no Sudeste que no Nordeste.

Em termos dos períodos de contração da renda, verifica-se que a recessão foi menos prejudicial aos indivíduos de menor renda em ambas as regiões, mas os efeitos adversos são menos intensos no Sudeste. Examinando, por fim, o período completo, pode-se constatar que a expansão da renda verificada ocorreu de forma mais que proporcional nas faixas mais baixas de renda nas duas regiões, porém com mais intensidade no Sudeste, para todos os índices. Essa evidência vai de encontro aos resultados anteriores que apontavam uma maior aproximação tanto em termos de renda familiar per capita como no índice de bem-estar de Sen entre as regiões. Entretanto, com essa nova medida, verifica-se que os ganhos de bem-estar na região Sudeste são mais intensos. Na seção seguinte procurar-se-á entender, a partir do comportamento do mercado de trabalho em ambas as regiões o que vem contribuindo para esse suposto distanciamento.

\section{DECOMPONDO OS GANHOS DE RENDA NO MERCADO DE TRABALHO ENTRE AS RE- GIÕES}

A literatura recente no Brasil, nessa área, tem procurado investigar quais os principais responsáveis pela redução da desigualdade de renda no país, especialmente a partir de 2001, quando se observa uma tendência consistente de queda como discutido na seção introdutória. Mais particularmente, os artigos de Hoffmann (2006) e Soares et alii (2006) têm se preocupado em calcular tanto a importância de cada componente de renda na renda familiar total como suas contribuições na trajetória de queda da desigualdade.

Utilizando Shorrocks (1982), que decompõe o índice de GINI em duas partes - o coeficiente de concentração de cada componente com relação à renda total e o peso de cada renda na renda total, esses autores mostraram que em média a renda de todos os trabalhos representa algo em torno de $75 \%$ da renda total, considerando o período 1995-2004. Ademais, em Hoffmann (2006), a contribuição desse

\footnotetext{
${ }^{2}$ A literatura tem usado com certa frequência essa forma de decomposição como pode ser atestada em Datt e Ravallion (1992), Kakwani (2000) e Son (2003).
} 
componente para a variação do índice de GINI entre 2001 a 2004 foi de quase $68 \%$ enquanto que em Soares et alii (2006), para um período mais extenso (1995-2004) a contribuição foi de 85\%.

Seguindo essas evidências, torna-se oportuno investigar o comportamento da renda do trabalho em cada região, já que a partir de alguns de seus componentes poderia estar a explicação para os diferenciais de bem-estar observado entre as regiões, seguindo a metodologia de Kakwani e Son (2008). Desse modo, seguindo Kakwani et alii (2006), pode-se mostrar que a taxa de crescimento renda do trabalho $\gamma\left(y_{1}\right)$ pode ser decomposta da seguinte forma:

$$
\gamma\left(y_{1}\right)=\gamma\left(e_{r}\right)+\gamma\left(h_{e}\right)+\gamma(\xi)+\gamma(\ell)
$$

onde $e_{r}$ é a razão entre o número de pessoas empregadas e o número de trabalhadores, $h_{e}$ o número de horas trabalhadas per capita, $\xi$ uma medida de produtividade dada pela razão entre $y_{L}$ e $h$, e $\ell$ a razão entre o número de trabalhadores (ocupados ou não) e o número de habitantes. É importante ter em mente que a decomposição acima proposta tem como finalidade a utilização de um artifício algébrico que possibilite a mensuração da influência que cada componente possui na evolução da renda do trabalho, e que desse modo, não se está sugerindo que essas variáveis estejam dissociadas, muito pelo contrário, sabe-se que há importantes relações entre elas.

A partir de (3) pode-se determinar a participação de cada variável na expansão/contração da renda do trabalho e, por conseguinte, o impacto na renda das regiões Nordeste e Sudeste. Os resultados da decomposição estão presentes nas Tabelas 3-5 a seguir. Na Tabela 3, é feita a decomposição para as populações como um todo para as regiões, enquanto que na Tabela 4 são consideradas as taxas anuais de crescimento apenas para os não-pobres e, finalmente, na Tabela 5, apenas para os indivíduos considerados pobres.

Tabela 3: Taxas anuais de crescimento da renda do trabalho per capita e fatores explicativos

\begin{tabular}{|c|c|c|c|c|c|c|c|c|c|c|}
\hline Ciclos renda & \multicolumn{2}{|c|}{ Renda } & \multicolumn{2}{|c|}{ Emprego } & \multicolumn{2}{|c|}{ Horas trabalhadas } & \multicolumn{2}{|c|}{ Força de trabalho } & \multicolumn{2}{|c|}{ Produtividade } \\
\hline 1995-1998 & $1.21 \%$ & $0.02 \%$ & $0.69 \%$ & $-0.14 \%$ & $-1.12 \%$ & $-0.80 \%$ & $0.56 \%$ & $0.29 \%$ & $1.08 \%$ & $0.67 \%$ \\
\hline 1999-2002 & $-0.36 \%$ & $0.31 \%$ & $1.31 \%$ & $0.31 \%$ & $-1.17 \%$ & $-0.20 \%$ & $0.00 \%$ & $1.24 \%$ & $-0.50 \%$ & $-1.04 \%$ \\
\hline 2003-2007 & $6.61 \%$ & $4.15 \%$ & $0.76 \%$ & $0.06 \%$ & $-0.84 \%$ & $0.33 \%$ & $0.69 \%$ & $0.90 \%$ & $6.00 \%$ & $2.86 \%$ \\
\hline \multicolumn{11}{|l|}{ Contração } \\
\hline 1998-1999 & $-6.27 \%$ & $-7.74 \%$ & $-1.38 \%$ & $-1.22 \%$ & $-0.22 \%$ & $0.18 \%$ & $2.53 \%$ & $1.94 \%$ & $-7.20 \%$ & $-8.64 \%$ \\
\hline $2002-2003$ & $-7.29 \%$ & $-7.82 \%$ & $0.31 \%$ & $-0.15 \%$ & $-0.80 \%$ & $-0.58 \%$ & $1.42 \%$ & $1.01 \%$ & $-8.22 \%$ & $-8.10 \%$ \\
\hline \multicolumn{11}{|l|}{$\begin{array}{l}\text { Período } \\
\text { completo }\end{array}$} \\
\hline $1995-2007$ & $1.29 \%$ & $0.17 \%$ & $0.67 \%$ & $-0.05 \%$ & $-0.94 \%$ & $-0.17 \%$ & $0.70 \%$ & $0.93 \%$ & $0.86 \%$ & $-0.53 \%$ \\
\hline
\end{tabular}

Como pode ser visto, no primeiro caso e para o período completo a produtividade é o principal responsável pela taxa de crescimento da renda do trabalho no Nordeste, especialmente advinda do período de expansão de 2003-2007, enquanto que no Sudeste, é a participação da força de trabalho. No caso das pessoas não-pobres (Tabela 4) é a participação da força de trabalho o principal componente que influencia a dinâmica da renda do trabalho em ambas as regiões. Ademais, pode-se ver que apesar do índice de produtividade ter crescido de forma expressiva na fase de expansão (2003-2007), observou-se grande queda neste indicador nas fases de contração, o que tornou suas taxas negativas para o período completo.

Entre as pessoas pobres, verifica-se que a taxa de crescimento da renda do trabalho foi negativa no Nordeste $(-0,45 \%)$ e positiva no Sudeste $(0,36 \%)$, no período completo. Na primeira região, o resultado foi motivado pela redução nas horas trabalhadas e na diminuição da participação na força de trabalho. Por outro lado, no Sudeste, foram os ganhos de produtividade nesse segmento da população que impulsionaram a renda do trabalho, com destaque ao expressivo aumento nos períodos de expansão, notadamente entre 2003-2007. 
Tabela 4: Taxas anuais de crescimento da renda do trabalho per capita e fatores explicativos, pessoas não pobres

\begin{tabular}{|c|c|c|c|c|c|c|c|c|c|c|}
\hline \multirow{2}{*}{$\begin{array}{l}\text { Ciclos renda } \\
\text { Expansão }\end{array}$} & \multicolumn{2}{|c|}{ Renda } & \multicolumn{2}{|c|}{ Emprego } & \multicolumn{2}{|c|}{ Horas trabalhadas } & \multicolumn{2}{|c|}{ Força de trabalho } & \multicolumn{2}{|c|}{ Produtividade } \\
\hline & & & & & & & & & & \\
\hline 1995-1998 & $0.45 \%$ & $-0.35 \%$ & $1.03 \%$ & $-0.14 \%$ & $-1.43 \%$ & $-0.83 \%$ & $0.44 \%$ & $0.31 \%$ & $0.41 \%$ & $0.30 \%$ \\
\hline 1999-2002 & $-1.24 \%$ & $0.21 \%$ & $0.31 \%$ & $0.19 \%$ & $-0.19 \%$ & $-0.03 \%$ & $0.93 \%$ & $1.40 \%$ & $-2.29 \%$ & $-1.35 \%$ \\
\hline 2003-2007 & $1.62 \%$ & $2.45 \%$ & $0.51 \%$ & $0.04 \%$ & $-0.80 \%$ & $0.11 \%$ & $-0.40 \%$ & $0.43 \%$ & $2.33 \%$ & $1.87 \%$ \\
\hline \multicolumn{11}{|l|}{ Contração } \\
\hline 1998-1999 & $-3.55 \%$ & $-7.10 \%$ & $-1.46 \%$ & $-1.07 \%$ & $0.49 \%$ & $0.26 \%$ & $2.59 \%$ & $2.05 \%$ & $-5.17 \%$ & $-8.34 \%$ \\
\hline $2002-2003$ & $-5.38 \%$ & $-6.26 \%$ & $0.10 \%$ & $-0.16 \%$ & $-0.42 \%$ & $-0.14 \%$ & $2.65 \%$ & $1.65 \%$ & $-7.70 \%$ & $-7.61 \%$ \\
\hline \multicolumn{11}{|l|}{$\begin{array}{l}\text { Período } \\
\text { completo }\end{array}$} \\
\hline $1995-2007$ & $-0.40 \%$ & $-0.33 \%$ & $0.39 \%$ & $-0.08 \%$ & $-0.67 \%$ & $-0.17 \%$ & $0.65 \%$ & $0.88 \%$ & $-0.77 \%$ & $-0.97 \%$ \\
\hline
\end{tabular}

Tabela 5: Taxas anuais de crescimento da renda do trabalho per capita e fatores explicativos, pessoas pobres

\begin{tabular}{|c|c|c|c|c|c|c|c|c|c|c|}
\hline \multirow{2}{*}{$\begin{array}{l}\text { Ciclos renda } \\
\text { Expansão }\end{array}$} & \multicolumn{2}{|c|}{ Renda } & \multicolumn{2}{|c|}{ Emprego } & \multicolumn{2}{|c|}{ Horas trabalhadas } & \multicolumn{2}{|c|}{ Força de trabalho } & \multicolumn{2}{|c|}{ Produtividade } \\
\hline & & & & & & & & & & \\
\hline 1995-1998 & $-0.81 \%$ & $0.11 \%$ & $0.01 \%$ & $-0.09 \%$ & $-0.56 \%$ & $-1.02 \%$ & $0.39 \%$ & $-0.89 \%$ & $-0.65 \%$ & $2.10 \%$ \\
\hline 1999-2002 & $-0.86 \%$ & $0.06 \%$ & $2.60 \%$ & $1.34 \%$ & $-2.66 \%$ & $-2.11 \%$ & $-1.66 \%$ & $-0.53 \%$ & $0.86 \%$ & $1.36 \%$ \\
\hline 2003-2007 & $0.26 \%$ & $1.33 \%$ & $1.04 \%$ & $1.32 \%$ & $-1.63 \%$ & $-1.33 \%$ & $-0.66 \%$ & $-2.07 \%$ & $1.51 \%$ & $3.41 \%$ \\
\hline \multicolumn{11}{|l|}{ Contração } \\
\hline 1998-1999 & $-0.07 \%$ & $-0.47 \%$ & $-0.58 \%$ & $-2.63 \%$ & $-1.56 \%$ & $0.29 \%$ & $3.55 \%$ & $3.23 \%$ & $-1.48 \%$ & $-1.36 \%$ \\
\hline $2002-2003$ & $-1.39 \%$ & $-1.11 \%$ & $0.73 \%$ & $-0.52 \%$ & $-1.25 \%$ & $-2.34 \%$ & $0.85 \%$ & $1.04 \%$ & $-1.72 \%$ & $0.71 \%$ \\
\hline \multicolumn{11}{|l|}{$\begin{array}{l}\text { Período } \\
\text { completo }\end{array}$} \\
\hline 1995-2007 & $-0.45 \%$ & $0.36 \%$ & $1.01 \%$ & $0.49 \%$ & $-1.58 \%$ & $-1.40 \%$ & $-0.17 \%$ & $-0.69 \%$ & $0.29 \%$ & $1.95 \%$ \\
\hline
\end{tabular}

\subsection{Os responsáveis pelos ganhos de produtividade no mercado de trabalho}

Visto que a produtividade foi um dos mais importantes componentes na evolução da renda do trabalho, especialmente no período de expansão de 2003-2007, é importante identificar quais foram os elementos principais que influenciaram esse desempenho nas duas regiões. Deste modo, seguindo novamente Kakwani et alii (2006), pode-se mostrar que:

$$
\gamma(\xi)=\gamma(s)+\gamma(\bar{r})
$$

em que a taxa de crescimento da produtividade $\gamma(\xi)$ pode ser decomposta em duas taxas: a da taxa média de escolaridade $\gamma(s)$ e a dos retornos médios por ano de estudo entre os trabalhadores $\gamma(\bar{r})$. As Tabelas 6-8 apresentam os resultados dessa nova decomposição para as regiões Nordeste e Sudeste, considerando também os períodos de expansão e contração da renda nacional, tanto para a população como um todo, como para os grupos de pobres e não pobres, semelhante ao que fora realizado anteriormente.

Inicialmente, como visto na Tabela 3 e reproduzido novamente na Tabela 6, houve um crescimento positivo na taxa anual de produtividade no Nordeste para todos os trabalhadores, mas negativo no Sudeste, isso considerando o período completo. Essa evidência pode ajudar a explicar o fato da renda familiar per capita no Nordeste ter crescido mais fortemente do que no Sudeste nesses anos. Apesar dos períodos de contração da renda terem apresentado forte redução na produtividade, o resultado positivo no Nordeste deveu-se especialmente ao período 2003-2007, com taxas de crescimento anuais de 6\%.

Atesta-se assim que os ganhos de produtividade partiram principalmente, do componente escolaridade com mais intensidade para o Nordeste. Por outro lado, exceto para o período 2003-2007, os 
Tabela 6: Taxas anuais de crescimento da produtividade e fatores explicativos

\begin{tabular}{lcccccc}
\hline Ciclos renda & \multicolumn{2}{c}{ Produtividade } & \multicolumn{2}{c}{ Escolaridade } & \multicolumn{2}{c}{ Retornos educação } \\
\hline & NE & SE & NE & SE & NE & SE \\
Expansão & & & & & & \\
$1995-1998$ & $1.08 \%$ & $0.67 \%$ & $2.35 \%$ & $2.52 \%$ & $-1.27 \%$ & $-1.85 \%$ \\
$1999-2002$ & $-0.50 \%$ & $-1.04 \%$ & $2.44 \%$ & $1.12 \%$ & $-2.93 \%$ & $-2.16 \%$ \\
$2003-2007$ & $6.00 \%$ & $2.86 \%$ & $2.09 \%$ & $1.13 \%$ & $3.90 \%$ & $1.73 \%$ \\
Contração & & & & & & \\
$1998-1999$ & $-7.20 \%$ & $-8.64 \%$ & $1.56 \%$ & $1.53 \%$ & $-8.76 \%$ & $-10.17 \%$ \\
$2002-2003$ & $-8.22 \%$ & $-8.10 \%$ & $3.10 \%$ & $1.98 \%$ & $-11.32 \%$ & $-10.08 \%$ \\
Período & & & & & & \\
completo & & & & & & \\
$1995-2007$ & $0.86 \%$ & $-0.53 \%$ & $2.28 \%$ & $1.58 \%$ & $-1.42 \%$ & $-2.11 \%$ \\
\hline Fonte: Elaboração dos autores a partir dos dados da PNAD/IBGE. & & & &
\end{tabular}

retornos da educação foram negativos para as duas regiões, com menor queda no Nordeste. Nas Tabelas 8-9 intensifica-se a investigação para os grupos de pessoas pobres e de não-pobres, respectivamente, em cada região.

Analisando a Tabela 7 , verifica-se que os ganhos de produtividade no Sudeste $(1,95 \%)$ foram bem superiores aos do Nordeste $(0,29 \%)$. Por sua vez, nos períodos de expansão, a produtividade entre os pobres no Sudeste cresceu muito mais do que no Nordeste, e nos períodos de retração da renda ela caiu menos ou continuou positiva, como ocorreu entre 2002-2003. Quanto aos elementos responsáveis pela produtividade, constata-se que a escolaridade entre os pobres, apesar de ter aumentado mais no Nordeste, seu retorno apresentou também uma maior queda no período, afetando assim a taxa como um todo na região no período completo. Nesse aspecto, Machado et alii (2007) e Machado e Ribas (2008) têm mostrado que a inserção no mercado de trabalho tem contribuído fortemente para que os indivíduos pobres melhorassem seus níveis de bem-estar e que, quanto mais intensa for a pobreza e mais tempo os indivíduos permaneçam nela, menores seriam suas chances de deixarem tal condição.

Essas conclusões podem nos ajudar a entender melhor os resultados acima, já que a pobreza no Nordeste tem sido mais aguda. Por fim, a Tabela 8 corrobora o desempenho verificado, na medida em que aponta que a produtividade entre os não-pobres caiu menos no Nordeste que no Sudeste, com maior avanço na taxa de escolaridade e menor queda do retorno da educação na primeira região.

Em resumo, os resultados encontrados apontam para a ideia de que, quando se avaliam a partir de 1995 os rebatimentos regionais do movimento nacional da renda observam-se dois movimentos assimétricos: nos períodos de expansão, a renda no Nordeste tem crescido de forma mais acelerada que no Sudeste e, nos períodos de contração, ela tem caído menos fortemente. A exceção é feita para o período recessivo de 2002-2003, em que a taxa de redução da renda no Nordeste é ligeiramente maior. Observase também para o índice de bem-estar de Sen, resultado semelhante ao movimento de aproximação da renda entre as regiões.

No entanto, quando se leva em conta o movimento de renda entre os mais pobres, através da construção de uma medida de intensidade de crescimento "pró-pobre", seguindo Kakwani e Son (2008), observa-se que nos períodos de expansão até 2002 há aproximação entre as regiões, mas após 2003, divergência. Nos períodos de contração da renda, não se verificou um padrão comum já que no primeiro período mostrou-se haver divergência enquanto que no segundo, aproximação. Como comentado an- 
Tabela 7: Taxas anuais de crescimento da produtividade e fatores explicativos, pessoas pobres

\begin{tabular}{lcccccc}
\hline Ciclos renda & \multicolumn{2}{c}{ Produtividade } & \multicolumn{2}{c}{ Escolaridade } & \multicolumn{2}{c}{ Retornos educação } \\
\hline & $\mathrm{NE}$ & $\mathrm{SE}$ & $\mathrm{NE}$ & $\mathrm{SE}$ & $\mathrm{NE}$ & $\mathrm{SE}$ \\
Expansão & & & & & & \\
$1995-1998$ & $-0.65 \%$ & $2.10 \%$ & $3.06 \%$ & $5.99 \%$ & $-3.71 \%$ & $-3.89 \%$ \\
$1999-2002$ & $0.86 \%$ & $1.36 \%$ & $4.20 \%$ & $2.77 \%$ & $-3.34 \%$ & $-1.41 \%$ \\
$2003-2007$ & $1.51 \%$ & $3.41 \%$ & $1.97 \%$ & $1.76 \%$ & $-0.46 \%$ & $1.65 \%$ \\
Contração & & & & & & \\
$1998-1999$ & $-1.48 \%$ & $-1.36 \%$ & $6.06 \%$ & $4.65 \%$ & $-7.54 \%$ & $-6.01 \%$ \\
$2002-2003$ & $-1.72 \%$ & $0.71 \%$ & $8.12 \%$ & $4.30 \%$ & $-9.84 \%$ & $-3.59 \%$ \\
Período & & & & & & \\
completo & & & & & & \\
$1995-2007$ & $0.29 \%$ & $1.95 \%$ & $3.65 \%$ & $3.52 \%$ & $-3.36 \%$ & $-1.57 \%$ \\
\hline Fonte: Elaboraçăo dos autores a partir dos dados da PNAD/IBGE. & & & &
\end{tabular}

Tabela 8: Taxas anuais de crescimento da produtividade e fatores explicativos, pessoas não pobres

\begin{tabular}{lcccccc}
\hline Ciclos renda & \multicolumn{2}{c}{ Produtividade } & \multicolumn{2}{c}{ Escolaridade } & \multicolumn{2}{c}{ Retornos educação } \\
\hline & $\mathrm{NE}$ & $\mathrm{SE}$ & $\mathrm{NE}$ & $\mathrm{SE}$ & $\mathrm{NE}$ & $\mathrm{SE}$ \\
Expansão & & & & & & \\
$1995-1998$ & $0.41 \%$ & $0.30 \%$ & $1.43 \%$ & $2.08 \%$ & $-1.02 \%$ & $-1.78 \%$ \\
$1999-2002$ & $-2.29 \%$ & $-1.35 \%$ & $1.33 \%$ & $0.95 \%$ & $-3.62 \%$ & $-2.30 \%$ \\
$2003-2007$ & $2.33 \%$ & $1.87 \%$ & $0.48 \%$ & $0.66 \%$ & $1.85 \%$ & $1.21 \%$ \\
Contração & & & & & & \\
$1998-1999$ & $-5.17 \%$ & $-8.34 \%$ & $1.74 \%$ & $1.52 \%$ & $-6.91 \%$ & $-9.86 \%$ \\
$2002-2003$ & $-7.70 \%$ & $-7.61 \%$ & $1.79 \%$ & $2.21 \%$ & $-9.49 \%$ & $-9.82 \%$ \\
Período & & & & & & \\
completo & & & & & & \\
$1995-2007$ & $-0.77 \%$ & $-0.97 \%$ & $1.14 \%$ & $1.29 \%$ & $-1.91 \%$ & $-2.26 \%$ \\
\hline Fonte: Elaboração dos autores a partir dos dados da PNAD/IBGE. & & & &
\end{tabular}


teriormente, a ausência de um padrão único pode ser devido ao fator que motivou a retração da renda nacional. Mas, avaliando o saldo no período completo, verifica-se uma tendência de distanciamento entre as regiões, com maiores ganhos na região Sudeste. Ademais, esse comportamento é explicado, em grande parte, porque a produtividade advinda da renda do trabalho dos mais pobres no Sudeste é mais intensa, devido especialmente ao melhor desempenho do retorno da educação entre essa parte da população.

\section{CONSIDERAÇÕES FINAIS}

Essa pesquisa contribui para a literatura nacional ao introduzir no debate sobre convergência de renda entre as regiões do país a ideia de convergência também de bem-estar. Essa discussão é colocada para os anos que seguem a partir da segunda metade da década de 1990 quando o Brasil inicia um longo período de estabilidade macroeconômica. Adicionalmente, a análise realizada é enriquecida com a ideia de se investigar os movimentos de renda e bem-estar nos períodos de expansão e contração da renda nacional.

O principal resultado observado é que apesar de se verificar uma contínua aproximação entre as regiões Nordeste e Sudeste em termos de renda familiar per capita e em termos de bem-estar medido pelo índice de Sen (1977), quando se pondera de forma mais intensa a renda dos mais pobres, através da medida de crescimento pró-pobre de Kakwani e Son (2008), verifica-se certo distanciamento entre as regiões. Isso ocorre principalmente porque os ganhos de produtividade no mercado de trabalho entre os pobres no Sudeste são bem mais intensos que no Nordeste, nesse período, o que acaba condicionando um padrão de geração com melhor distribuição na primeira região. Em extensão a esse resultado, observa-se também que apesar do aumento da escolaridade entre os pobres no Nordeste ter sido superior ao Sudeste, seu retorno foi menor, o que contribuiu para uma menor produtividade no agregado. Evidências interessantes nessa direção já tinham sido apontadas por Resende e Wyllie (2006).

Uma extensão natural dessa pesquisa é procurar entender melhor os condicionantes que expliquem porque a produtividade dos pobres tem crescido mais no Sudeste. A estrutura educacional familiar pode ser um importante caminho de novas investigações, como observado em Ramos e Reis (2008). Outro ponto a ser explorado é compreender melhor como o mercado de trabalho busca e remunera trabalho qualificado entre as regiões já que os principais estudos existentes olham apenas para a questão do ponto de vista nacional. Ramos (2006), por exemplo, conclui que o comportamento dos retornos de educação deve-se em grande parte à natureza das mudanças na oferta e demanda por qualificação no âmbito do mercado de trabalho. Na mesma direção, Andrade e Menezes-Filho (2005) mostram que, embora havendo redução na proporção de pessoas com baixa escolaridade na força de trabalho do Brasil, a demanda relativa por trabalho qualificado e intermediário não cresceu entre 1981 e $1999 .{ }^{3}$ Nesse sentido, as estimativas por regiões seriam extensões interessantes e ajudariam a melhor compreensão da questão do desequilíbrio regional brasileiro.

Ademais, além desses pontos já levantados, novos estudos poderiam ressaltar também os aspectos qualitativos do serviço de educação praticado em cada região do país, pois, a dificuldade que as pessoas têm de transformar educação em renda depende também do tipo de educação recebida, cuja qualidade revela-se no mercado de trabalho. Nesse sentido, a participação do setor público como provedor de educação, especialmente em regiões pobres, tem um papel extremamente relevante. Como observado por Sachsida et alii (2004) as pessoas de baixa renda estariam sempre propensas em não investir em educação dado o elevado custo de oportunidade até que o investimento realizado se torne atrativo. Por fim, de um modo geral essa pesquisa abre também novos campos de investigação a partir da revelação dos comportamentos distintos dos diversos componentes da renda do trabalho tanto no que se refere

\footnotetext{
${ }^{3}$ Seria interessante como extensão a esta pesquisa, a ampliação do período analisado para a década de 2000 , abrangendo a recuperação do mercado de trabalho no Brasil a partir de 2003.
} 
às regiões analisadas como entre as pessoas pobres e não-pobres. Ajudar a explicar os padrões distintos verificados contribuirá de sobremaneira para a literatura sobre desequilíbrio regional no Brasil.

\section{BIBLIOGRAFIA}

Adams, R. H. J. (2004). Economic growth, inequality and poverty: Estimating the growth elasticity of poverty. World Development, 32(12):1989-2014.

Andrade, A. A. S. \& Menezes-Filho, N. (2005). O Papel da Oferta de Trabalho no Comportamento dos Retornos à Educação no Brasil. IBMEC, São Paulo.

Andrade, E., Laurini, M., Madalozzo, R., \& Pereira, P. L. V. (2004). Convergence clubs among Brazilian municipalities. Economics Letters, 83:179-184.

Barros, R. P. (1993). Regional disparities in education within Brazil: The role of quality of education. IPEA, Texto para Discussão 311, Brasília.

Barros, R. P., Camargo, J. M., \& Mendonça, R. (1997). A estrutura do desemprego no Brasil. IPEA, Texto para Discussão 478, Brasília.

Barros, R. P., Carvalho, M., Franco, S., \& Mendonça, R. (2006). A queda recente da desigualdade de renda no Brasil. In Barros, E. P., Foguel, M. N., \& Ulyssea, G., editors, Desigualdade de Renda no Brasil: Uma Análise da Queda Recente, chapter 2. IPEA, Brasília.

Barros, R. P. \& Mendonça, R. (1995). Os determinantes da desigualdade no Brasil. IPEA, Texto para Discussão 377, Brasília.

Bourguignon, F. (2004). The Poverty-Growth-Inequality Triangle. World Bank, Washington, DC.

Corseuil, C. H. \& Foguel, M. N. (2002). Uma sugestão de deflatores para rendas obtidas a partir de algumas pesquisas domiciliares do IBGE. IPEA, Texto para Discussão 897, Rio de Janeiro.

Datt, G. \& Ravallion, M. (1992). Growth and redistribution component of changes in poverty measures: A decomposition with applications to Brazil and India in the 1980s. Journal of Development Economics, 83:275-95.

Ellery Jr., R. \& Ferreira, P. (1996). Convergência entre a renda per capita dos estados brasileiros. Revista de Econometria, 16(1):83-103.

Ferreira, A. \& Diniz, C. (1995). Convergência entre as rendas per capita estaduais no Brasil. Revista de Economia Política, 15(4):38-56.

Ferreira, A. H. B. (1996). Evolução recentes da renda per capita estaduais no Brasil: O que a nova evidência mostra. Revista Econômica do Nordeste, 27(3):363-374.

Ferreira, A. H. B. (1999). Concentração regional e dispersão das rendas per capita estaduais: Um comentário. Estudos Econômicos, 29(1):47-63.

Ferreira, A. H. B. (2000). Convergence in Brazil: Recent trends and long-run prospects. Applied Economics, 32:479-489.

Gondim, J. L., Barreto, F. A., \& Carvalho, J. R. (2007). Condicionantes de clubes de convergência no Brasil. Revista Estudos Econômicos, 37(1):71-100. 
Hoffmann, R. (2006). Transferências de renda e a redução da desigualdade no Brasil e 5 regiões entre 1997 e 2004. Mimeografado.

IPEA (2008). PNAD 2007: Primeiras análises, pobreza e mudança social, volume. Instituto de Pesquisas Econômicas Aplicada. Comunicado da Presidência 9. Brasília.

Jones, C. I. (1997). On the evolution of the world income distribution. Journal of Economic Perspectives, 11(3):19-36.

Kakwani, N. (2000). On measuring growth and inequality components of poverty with application to Thailand. Journal of Quantitavive Economics, 16:67-68.

Kakwani, N., Neri, M., \& Son, H. (2006). Linkages between growth, poverty and the labor market. Fundação Getulio Vargas, Ensaios Econômicos 634, Rio de Janeiro.

Kakwani, N. \& Son, H. H. (2008). Poverty equivalent growth rate. The Review of Income and Wealth, 54(4):643-55.

Machado, A. F. \& Ribas, R. P. (2008). Mudanças no mercado de trabalho retiram famílias da pobreza? Determinantes domiciliares e agregados para a saída da pobreza nas regiões metropolitanas do Brasil. IPEA, Texto para Discussão 1336, Brasília.

Machado, A. F., Ribas, R. P., \& Penido, M. (2007). Mobilidade entre os estados de pobreza e inserção no mercado de trabalho: Uma análise para o Brasil metropolitano em 2004. Economia Aplicada, 11(2):253-279.

Marinho, E., Soares, F., \& Benegas, M. (2004). Desigualdade de renda e eficiência técnica na geração de bem-estar entre os estados brasileiros. Revista Brasileira de Economia, 58(4):583-608.

Mossi, M. B., Aroca, P., \& Fernandes, I. J. (2003). Growth dynamics and space in Brazil. International Regional Science Review, 26(3):393-418.

Neri, M. C. (2006). Desigualdade, estabilidade e bem-estar social. In Barros, R. P., editor, Desigualdade de Renda no Brasil: Uma Análise da Queda Recente. IPEA, Brasília.

Neri, M. C. (2007). Miséria, desigualdade e políticas de renda: O Real do Lula. FGV/IBRE, CPS, Rio de Janeiro.

Pessoa, S. (2001). Existe um problema de desigualdade regional no Brasil? In Anais do Encontro Nacional de Economia, Salvador. ANPEC.

Pessoa, S., Cavalcanti, P., \& Schymura, L. G. (2007). Por que o Brasil não precisa de política industrial? EPGE/FGV, Ensaios Econômicos 644, Rio de Janeiro.

Ramos, L. (2006). Desigualdade de rendimentos do trabalho no Brasil no período pós-real. IPEA, Nota Técnica, Rio de Janeiro.

Ramos, L. \& Reis, M. C. (2008). A escolaridade dos pais e os retornos à educação no mercado de trabalho. IPEA, Nota Técnica, Rio de Janeiro.

Ravallion, M. (2001). Growth, inequality and poverty: Looking beyond averages. World Development, 29(11):1803-1815.

Resende, M. \& Wyllie, R. (2006). Retornos para educação no Brasil: Evidências empíricas adicionais. Economia Aplicada, 10(3):349-365. 
Sachsida, A., Loureiro, P. R. A., \& Mendonça, M. J. C. (2004). Um estudo sobre retorno em escolaridade no Brasil. Revista Brasileira de Economia, 58(2):249-265.

Salvato, M., Duarte, A., \& Cavalcanti, P. (2007). Disparidades regionais ou educacionais? Um exercício com contrafactuais. Mimeografado.

Sen, A. K. (1977). On Economic Inequality. Clarendon Press, Oxford.

Shorrocks, A. (1982). Inequality decomposition by factor components. Econometrica, 50:193-211.

Soares, F. V., Soares, S., Medeiros, M., \& Guerreiro, R. (2006). Programas de transferência de renda no Brasil: Impactos sobre a desigualdade. IPEA, Texto para Discussão 1228, Brasília.

Son, H. H. (2003). A new poverty decomposition. Journal of Economic Inequality, 1(2):181-187.

Son, H. H. (2007). Interrelationship between growth, inequality and poverty: The Asian experience. Asian Development Review, 24(2):37-63.

Wal, P. A. (2008). Economic growth and poverty reduction: Evidence from Kazakhstan. Asian Development Review, 24(2):90-115.

Wodon, Q. \& Yitzhaki, S. (2005). Growth and convergence: A social welfare framework. Review of Income and Wealth, 51(3):443-454.

Zini Jr., A. (1998). Regional income convergence in Brazil and its socio-economic determinants. Economia Aplicada, 2:383-411. 